\title{
Production and test of Micromegas boards for the ATLAS New Small Wheel project
}

\author{
Luigi Longo, on behalf of the ATLAS Muon Collaboration ${ }^{a, *}$ \\ ${ }^{a}$ CERN, \\ Esplanade des Particules 1, 1211 Meyrin, Switzerland \\ E-mail: luigi.longo@cern.ch
}

\begin{abstract}
The muon system of the ATLAS Experiment will be upgraded at the end of the 2019-2021 LHC shutdown with Micromegas detectors covering an active area of about $1280 \mathrm{~m}^{2}$, being the largest system based on Micro Pattern Gaseous Detector (MPGD) ever built so far. The key element of the detectors are the anode boards which carry the readout strips, the resistive protection layer and the insulating pillars supporting the mesh. In total 32 different types of boards, for 136 modules, with size up to $40 \times 220 \mathrm{~cm}^{2}$ have been produced. The boards are produced by two companies in Europe, which opened the road to MPGD mass production, with the production now finished. The boards undergo a detailed quality control and quality assurance checks at CERN, prior to being mounted into the final detectors. In this work the technological transfer effort made by CERN and ATLAS to make the Micromegas board production an industrial process is described.
\end{abstract}

40th International Conference on High Energy physics - ICHEP2020

July 28 - August 6, 2020

Prague, Czech Republic (virtual meeting)

\footnotetext{
${ }^{*}$ Speaker
} 


\section{Introduction}

During the 2019-2021 Large Hadron Collider (LHC)[1] shutdown, the ATLAS experiment [2] is upgrading both of its innermost forward muon stations (small wheels) within the so-called New Small Wheel project [3]. The upgrade is based on the small-strip Thin Gap Chambers (sTGC) and resistive Micromegas (MM) technologies and is designed to operate in the harsh environment expected for High-Luminosity LHC. Each of the New Small Wheels consists of 8 small and 8 large overlapping sectors, each of which is made of 2 Micromegas and 2 sTGC wedges. Each MM wedge is in particular made of 2 modules and each module consists of 4 layers of Micromegas detector; the first two layers reconstruct the precision coordinate (Eta-layers), while the last 2 (the stereo layers) the second coordinate.

Depending on the height of the module, each MM layer has the anode plane composed of 5 or $3 \mathrm{PCBs}^{1}$, i.e. readout boards (or anode boards), as shown in Figure 1a. Each readout board is made of a FR4 layer, on which the copper readout strips lie, and of a resistive protection layer, composed of a Kapton ${ }^{\circledR}$ insulating layer on which the resistive strips are screen-printed with a strip pitch of $425 / 450 \mu \mathrm{m}$ for small/large module. The FR4 and Kapton ${ }^{\circledR}$ are glued together under specific conditions of temperature and pressure. Finally, on top of the resistive strips, the pillars holding the micromesh are developed. A schematic view of an anode board is reported in Figure 1b.

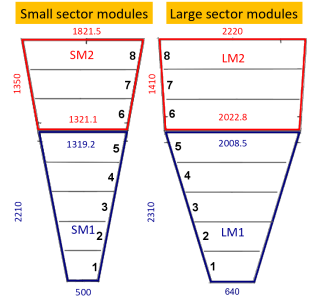

(a)

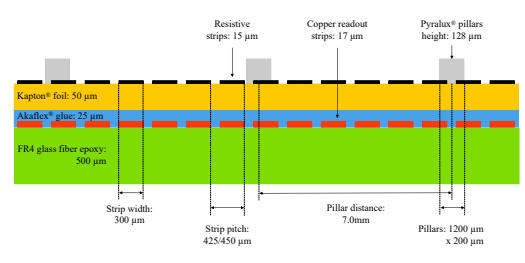

(b)

Figure 1: (a) Schematic view of the subdivision applied to the readout plane for small and large sectors. Each sector is divided in 2 trapezoidal modules with different dimensions and 8 PCBs are needed to cover the full readout plane (all units are in $\mathrm{mm}$ ) [4]. (b) Schematic view of the NSW Micromegas readout board with the dimensions of each component.

While the resistive layers are produced in Japan at Matsuda Screen Inc., the readout boards are manufactured by 2 PCB companies, ELTOS SpA (Italy) and ELVIA PCB Group (France). In order to fit the large (L) and small (S) modules dimensions and to produce eta (E) and stereo (S) layers, 32 different types ${ }^{2}$ of readout boards have been produced, for a total production of about 3000 boards. Several actions have been taken in the PCB companies to guarantee a final product within the specifications, as for example: the rescaling of the board dimensions to take into account the FR4 expansion $^{3}$ due to moisture uptake, with the additional request to the companies to wait a 4-week

${ }^{1}$ The PCB industry usually can handle PCB dimensions of $600 \mathrm{~mm}$ in the short dimension and up to $2500 \mathrm{~mm}$ in the large dimension. In order to stay within the dimensions that most PCB manufacturers can handle, readout board size has been kept in one dimension below $600 \mathrm{~mm}$.

${ }^{2}$ Each type of readout board is labelled following the acronym type of module, type of layer and PCB number (from Figure 1a). For example SE1: Small sector, Eta Layer, PCB 1.

${ }^{3}$ The FR4 expansion is about $400 \mu \mathrm{m} / \mathrm{m}$. 
period to let the board expand before the final cutting and drilling; the application of dedicated surface treatments (pumice treatment, brushing) for having a rougher surface, which ensures a good pillar adhesion; the tuning of temperature and pressure during the gluing step to guarantee a final surface resistivity within the specifications (average resistivity between 0.28 and $2.6 \mathrm{M} \Omega / \mathrm{sq}$ ).

\section{Quality control and quality assurance checks and results}

The readout boards undergo a detailed quality control and quality assurance (QAQC) checks at CERN, reaching an acceptance yield of $84 \%$. Each of the PCB is indeed visually inspected for any possible defect, such as dent, pit, bump, and it goes under several tests with the aim of evaluate its electrical properties (as for example, insulation between resistive and readout strips or between the surface holding the mesh and the HV distribution line), the alignment and rotation between resistive pattern and copper strips, the accuracy of cutting and milling, the possible elongation with respect to the nominal dimensions, the lack of pillars and the possible inhomogeneity in their height, the final surface resistivity. All these aspects play in fact a crucial role in the detector performance: the board dimensions are for example important for a precise tracking, while the height of the pillars and the resistivity are fundamental for the detector high-voltage stability. Focusing in particular on these last aspects, it has been observed that most of the boards have an elongation with respect to the nominal dimensions within the specifications (Figure 2a), the pillar height is homogeneous within $5 \mu \mathrm{m}$ for each PCB and on average a difference of $2 \mu \mathrm{m}$ is present between ELTOS $(\sim 121 \mu \mathrm{m})$ and ELVIA $(\sim 119 \mu \mathrm{m}$ ) boards (Figure $2 \mathrm{~b}$ ), the change in superficial resistivity during the gluing step or the surface treatment is negligible for those resistive foils with an initial resistivity lower than $1 \mathrm{M} \Omega /$ sq (Figure 2c).

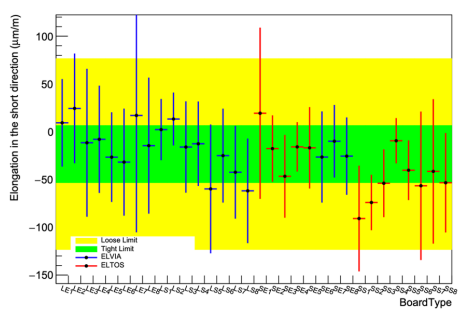

(a)

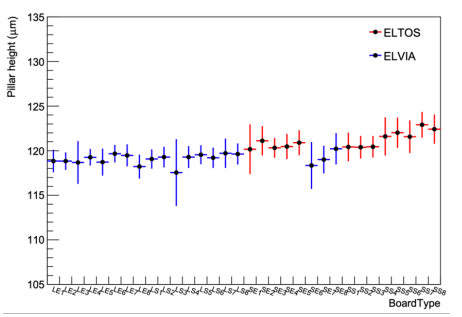

(b)

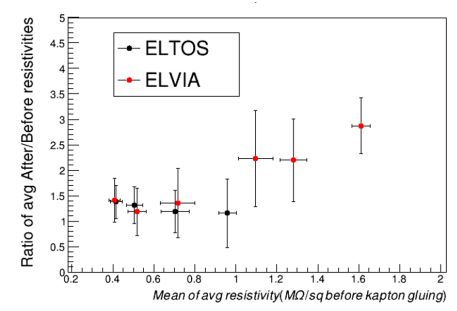

(c)

Figure 2: (a) Elongation along the short PCB direction and (b) average pillars height per board type; (c) scatter plot of the final over initial superficial resistivity ratio as a function of the initial value.

\section{References}

[1] L. Evans and P. Bryant (editors) 2008 JINST 3 S08001.

[2] ATLAS Collaboration, 2008 JINST 3 S08003.

[3] T. Kawamoto et al., CERN-LHCC-2013-006, ATLAS-TDR-020.

[4] P. Lösel and R. Müller, 2015, arXiv: 1508.02541 [physics.ins-det] 